\title{
Intestinal parasitic infections and associated factors among mentally disabled and non- disabled primary school students, Bahir Dar, Amhara regional state, Ethiopia, 2018: a comparative cross-sectional study
}

\author{
Agumas Ayalew Fentahun ${ }^{1 *}$ (D) Anemaw Asrat ${ }^{2}$, Abebayehu Bitew² and Selamawit Mulat ${ }^{3,4}$
}

\begin{abstract}
Background: Intestinal parasitic infections are still common in low-income countries including Ethiopia, particularly in children due to low-quality drinking water, poor personal and environmental sanitation. Disabled individuals are excluded from most academic, economic, social and cultural opportunities, they are among the poorest and most marginalized of the whole world's people.

The aim of this study was to assess the prevalence of intestinal parasitic infections and associated factors among mentally disabled and non-disabled students at primary schools in Bahir Dar city, Amhara regional state, Ethiopia, 2018.

Methods: A school-based Comparative cross-sectional study design was conducted from November 1-30, 2018. A total of 418 study participants, 104 mentally disabled and 314 non-disabled students were recruited through a simple random sampling technique. The collected data were coded, entered and cleaned with EpiData version 3.1 and analyzed using SPSS version 23. Multivariable logistic regression was conducted to identify factors associated with intestinal parasitic infections. The adjusted odds ratio with a 95\% Confidence interval at a 5\% level of significance was used to measure the strength of association.

Results: The mean age of study participants was $14.05 \pm 3.66$ and $11.96 \pm 2.94$ for mentally disabled students and nondisabled students. Prevalence of parasitic infection was $56.70 \%(n=59)$ for mentally disabled students whereas $41.10 \%(n=129)$ for non-disabled students. Unclean fingernails [AOR $=2.42 ; 1.40,4.17]$, health checkups [AOR $=1.87 ; 1.16$, 3.02], hand washing with water only $[A O R=2.48 ; 1.49,4.12]$, cooking and sanitation source of water $[A O R=4.40 ; 2.32$, 8.36], Grade [ (1-4)] $[\mathrm{AOR}=2.27 ; 1.41,3.67]$, sex $[\mathrm{AOR}=1.64 ; 1.03,2.63]$ and Family size $>=7[\mathrm{AOR}=2.74 ; 1.25,5.99]$ were variables which showed statistically significant association with intestinal parasitic infections.

Conclusion: The prevalence of intestinal parasitic infection was higher among mentally disabled students than nondisabled students. Unclean fingernails, health checkups, hand washing habits, source of water, family size, sex and Grade of students have had a statistically significant association with intestinal parasitic infections. Periodic medicinal treatment was needed twice a year for mentally disabled and once a year for non-disabled students.
\end{abstract}

Keywords: Mental disability, Intestinal parasitic infection, Primary school students

\footnotetext{
*Correspondence: fentahun143@gmail.com; agumlt@yahoo.com

${ }^{1}$ Family Guidance Association of Ethiopia, Bahir Dar Model Sexual and

Reproductive Health Clinic, Bahir Dar, Ethiopia

Full list of author information is available at the end of the article
}

(c) The Author(s). 2019 Open Access This article is distributed under the terms of the Creative Commons Attribution 4.0 International License (http://creativecommons.org/licenses/by/4.0/), which permits unrestricted use, distribution, and reproduction in any medium, provided you give appropriate credit to the original author(s) and the source, provide a link to the Creative Commons license, and indicate if changes were made. The Creative Commons Public Domain Dedication waiver (http://creativecommons.org/publicdomain/zero/1.0/) applies to the data made available in this article, unless otherwise stated. 


\section{Background}

Parasites are defined as organisms that get food and shelter from other organisms or the host and often harm it. For a parasitic to be outlined as intestinal, it must have an intestinal life cycle stage. Additionally, it may have a life cycle stage in the heart, circulation, lung, tissue, and other animals on the surrounding [1]. The most routes of entry of intestinal parasites into the human body are ingestion, skin penetration, aspiration, and auto-infection. Ingestion (fecal-oral routes); contamination of water and food is generally the foremost style of transmitting protozoan infections [2].

Intestinal parasitic infections (IPIs) comprise both helminths and protozoan which form the most common infections worldwide [3]. Helminths are worms with many cells while protozoa are parasites which have only one single cell and can multiply inside the human body [4] Most frequent Intestinal helminths were A. lumbricoides, E. vermicularis, Hymenolepis species, T. trichiura, E. vermicularis, S. stercoralis, Hookworm, S. mansoni, and Tania species, while most frequent intestinal protozoa include E. histolytic and G. intestinalis [5].

Globally, IPIs remains endemic. About one-third of the world's population, more than 2 billion people are infected by IPIs, with the most being children $[6,7]$. Similarly, in South Africa, primary school children of Eastern Cape Province $64.8 \%$ study participants were positive for ova and cysts of parasites from which $57.4 \%$ were known pathogenic parasites [8]. Among students in Kigali, Rwanda More than half $50.5 \%$ of the stools examined were infected with an intestinal parasite [9]. In Ethiopia too, there were different studies conducted in different regions at different time periods to assess the PIPIs; of which in Gamo area prevalence of intestinal parasitic infections was 39.9\% [10] while in Chencha town $81.0 \%$ [11].

Disability is the umbrella term for impairments, activity limitations and participation restrictions, pertaining to the negative aspects of the interaction between a personal (with a health condition) and that individual's contextual factors [12]. They were routinely excluded from most academic, economic, social and cultural opportunities, they're among the poorest and most marginalized of the world's young people [13]. Due to the above factors, improper sanitary hygiene and illiteracy about personal hygiene People with disabilities have a higher risk of IPIs than non-disabled individuals [14].

Although they have a higher risk of IPIs due to self and family care problems, there were no published studies on the burden of IPIs among people with disabilities in Ethiopia. Therefore, the aim of this study was to assess the prevalence of Intestinal parasitic infections and associated factors among mentally disabled and non-disabled students in Bahir Dar city at primary schools' students.

\section{Methods}

\section{Study area}

The study was conducted at Bahir Dar city among students at public primary schools which contain mentally disabled and non-disabled students inclusively. Bahir Dar was one of the fast-growing cities in the country and serves as capital city of Amhara regional state which located $563 \mathrm{~km}$ away from the North West of Addis Ababa, the capital city of Ethiopia, having a total Area of $28 \mathrm{~km}^{2}$, latitude and longitude of $11^{\circ} 36^{\prime} \mathrm{N} 37^{\circ} 23^{\prime} \mathrm{E}$ and an elevation of $1840 \mathrm{~m}(6,040 \mathrm{ft}$. feet) above sea level. The city had four hospitals ( 2 private), ten health centers and different private profitable and non-profitable health institutions. Primary schools in Bahir Dar city have not sufficient latrine lavatories and poor in environmental sanitation. In Bahir Dar, mentally disabled students registered well and attained their education as special needs at primary schools however still they are at first cycle primary schools due to their mental disability problem. So, Bahir Dar city was the real setting to conduct this comparative study among mentally disabled and non-disabled primary school students. According to Bahir Dar city Educational office, there were 122 primary school students registered as mentally disabled and 6816 non-disabled primary school students attending in those four primary schools [15]. A school-based comparative cross-sectional study design was conducted from November 1 to November 30, 2018.

\section{Study population}

Mentally disabled students: students registered as had being an intellectual problem at primary schools during the study period.

Non-disabled students: Students who had not any form of disability and attending primary schools during the study period.

\section{Sample size determination and sampling technique}

The required final sample size was calculated using double population proportion formula: Two groups have unequal sample size (1:3, Disabled to nondisabled ratio), with the assumptions of $95 \%$ confidence level, power of $80,5 \%$ of marginal error, $10 \%$ non-response rate, and prevalence of IPIs among non-disabled students $(\mathrm{P} 2=65.5 \%)$, from the previous study conducted in Bahir Dar city, Dona Berber primary school students [16] and the prevalence of IPIs among mentally retarded students in the previous study is not known; Therefore we took $\mathrm{P} 1=50 \%$ as prevalence for disabled students based on the assumption of getting greater sample size with $p=50 \%$. Therefore, the final required sample size was 458 (344 non-disabled students and 114 mentally Disabled students) were enrolled in the study using a simple random sampling technique. 
First, the total number of disabled and non-disabled students were obtained from each selected school. Second, the required sample sizes of both mentally disabled and non-disabled students were distributed independently among the selected schools proportionally based on the total number of students in each school. Then, the number of students required to be enrolled was allocated proportionally based on grade level.

Finally, study participants were selected by simple random sampling technique (Random Number Generator) using a list of the students as a sampling frame. Students' were identified, and their parents contacted by teachers or children. Accordingly, 44, 11, 43 and 16 disabled students and 146, 52, 12 and 134 non-disabled students were sampled from Shibit, Yekatit 23, Felege Abay and Teyma primary schools.

\section{Operational definitions}

Ova or Parasite seen/positive/: One of the parasites, eggs, cyst, trophozoite, larva or more than one of this diagnostic stage of intestinal parasites was found in study participants stool sample.

Water contact: a student who has playing, swimming, fishing and/or irrigation experience on the various source of water (like lakes, rivers, ponds).

Mentally Disabled: students registered as having an intellectual problem in the selected schools or students who attained their education as special needs due to mental impairment will be considered as mentally Disabled students.

Pit latrine is a type of toilet that collects human feces in a hole in the ground.

Flush toilet is a toilet that disposes of human excreta (urine and feces) by using water to flush it through a drainpipe to another location for disposal, thus maintaining a separation between humans and their excreta.

A Pour-flush toilet is like a regular cistern flush toilet except that the water is poured in by the user, instead of coming from the cistern above.

\section{Data collection tools and procedures}

The data for this study were collected from November 1 to November 30, 2018, using both a structured questionnaire adopted from different kinds of literature and for laboratory data with direct stool microscopy. Four data collectors (2 clinical Nurses for the collection of data related to the associated factor and 2 laboratory technicians for direct stool microscopy) and two supervisors (one health officer and one laboratory technologist) were assigned. One day prior to our cross-sectional parasitological and questionnaire surveys a written informed consent form for the parent/guardian of participating children were left with the teachers and distributed to eligible students' parents/guardians through students or through teachers. During the school-based survey, the signed informed consent sheets were cheeked, unique identification numbers assigned to each participating student and the same number of plastic bottles (stool cup) given for stool collection. A short interview held with each student/parent/guardian, using a questionnaire pertaining to hygiene behavior, drinking water and sanitation. After completing the interview, the participants were asked to give one thumb size of a stool sample and orientation given how to collect the stool sample for each participant, and stool samples were collected.

Laboratory technicians did a stool microscopic examination soon (within $30 \mathrm{~min}$ ) not to miss the trophozoite of protozoa by using a direct wet mount [17]. The rest samples were preserved with $10 \%$ formalin to process using the formol-ether concentration technique [17].

In Bahir Dar Model Sexual and Reproductive Health Clinic Laboratory, $10 \%$ formalin, Ether, distilled water/ normal saline, cone-shaped test tubes, Microscope, and centrifuge were used to process stool samples. Finally, left-over samples were decontaminated with appropriate bleach and discarded to Bahir Dar Model Sexual and Reproductive Health Clinic incinerator.

\section{Data management/processing and analysis procedures}

Data were coded, entered and cleaned, using EpiData version 3.1 and exported to Statistical package for social science (SPSS) version 23 for analysis. Frequencies and percentages were generated. Tables and graphs used for data presentation. The bi-variable logistic regression method was used to select candidate variables. Independent variables resulting in a $p$-value of less than 0.2 on bi-variable analysis were considered in the multivariable logistic regression analysis for further analysis. Multivariable logistic regression with the forward method was carried out to identify factors associated with IPIs. Adjusted odds ratio (AOR) with 95\% Confidence interval (CI) at 5\% level of significance was used to measure the strength and significance of the association. Hosmer and Lemeshow goodness of fit test was used for checking the logistic regression model fitting assumption, the assumption was fulfilled at $x^{2}=7.809$ and $P$ value $=0.452$ since it was $>0.05$.

\section{Results}

Socio-demographic characteristics of the respondents A total of 418, 104 mentally disabled and 314 nondisabled primary school students participated in the study with 91.23 and $91.28 \%$ response rate for disabled and nondisabled students respectively. Of which females account $41.30 \%(n=43)$ from mentally disabled students and $55.10 \%(n=173)$ from non-disabled students. The mean 
age of study participants was $14.05 \pm 3.66$ and $11.96 \pm 2.94$ for mentally disabled students and non-disabled students respectively [Table 1].

\section{Student's hygiene and safety-related factors}

Regards to student's hygiene and safety $55.80 \%(n=58)$ of mentally disabled students and $24.20 \%(n=76)$ nondisabled students fingernails had dirty materials. Above half of the mentally disabled students, $52.90 \%(n=55)$ had washed their hands sometimes before food while 93.30\% $(n=293)$ of non-disabled students did wash their hands always before food [Table 2].

Concerning the availability of latrine in the home, all of them (mentally disabled and non- disabled) students said that they had a latrine at their compound. According to the information provided, $65.40 \%(n=68)$ mentally disabled and $52.50 \%(n=165)$ non-disabled primary school students had not undergone health checkups for over a year [Table 3].

\section{Food and drink establishments' related factors}

In relation with drinking water supply in the home, all of them (mentally disabled and non-disabled students received from tape water while about $9.60 \%(n=10)$ of the mentally disabled and $20.70 \%(n=65)$ of nondisabled students' households cooking and sanitation water source were from both tape water and well water [Table 4].

\section{Prevalence of intestinal parasitic infections}

Above half of the mentally disabled students, $56.70 \%$ $(n=59)$ (95\% CI: 47.20-66.20) were infected with at least a single intestinal parasite. While, about $41.10 \%$ ( $n=129)$ (95\% CI: 35.70-46.00) of non-mentally disabled students were infected with intestinal parasites; whereas the overall prevalence of intestinal parasitic infections was $45 \%(n=188)$ (95\% CI: 40.20-49.80). Point estimate for the difference of the two populations was $15.60 \%$ with $95 \% \mathrm{CI}=(0.05,0.27)$ [Fig. 1].

Table 1 Socio-Demographic Characteristics of mentally disabled and non-disabled primary school students' and their families in Bahir Dar City, Amhara Regional State, Ethiopia, $2018(n=418)$

\begin{tabular}{|c|c|c|c|c|c|c|c|}
\hline \multicolumn{2}{|l|}{ Characteristics } & \multicolumn{6}{|c|}{ Students with disability status } \\
\hline & & \multicolumn{2}{|c|}{ Disabled students $(n=104)$} & \multicolumn{2}{|c|}{ Non-disabled students $(n=314)$} & \multicolumn{2}{|c|}{$\operatorname{Total}(n=418)$} \\
\hline Variables & Category & Freq (\%) & $x^{2}, p$ & Freq (\%) & $x^{2}, p$ & Freq (\%) & $x^{2}, p$ \\
\hline \multirow[t]{2}{*}{ Mental disability } & Yes & 104(100) & - & 0 & - & 104(24.9) & $X^{2}=7.730, P=0.005$ \\
\hline & No & 0 & & $314(100)$ & & $314(75.1)$ & \\
\hline \multirow[t]{3}{*}{ Age } & $<=9$ & $15(14.4)$ & $x^{2}=9.368, p=0.009$ & $88(28.0)$ & $X^{2}=12.608, P=0.002$ & 103(24.6) & $X^{2}=11.317, P=0.003$ \\
\hline & $10-14$ & $38(36.5)$ & & 151(48.1) & & 189(45.2) & \\
\hline & $>=15$ & $51(49.0)$ & & $75(23.9)$ & & $126(30.1)$ & \\
\hline \multirow[t]{2}{*}{ Sex } & Male & $61(58.7)$ & $x^{2}=0.926, p=0.336$ & $141(44.9)$ & $X^{2}=4.378, P=0.036$ & 202(48.3) & $X^{2}=6.692, P=0.010$ \\
\hline & Female & $43(41.3)$ & & 173(55.1) & & $216(51.7)$ & \\
\hline \multirow[t]{4}{*}{ Religion } & Orthodox & $81(77.9)$ & $X^{2}=1.043, p=0.791$ & $234(74.5)$ & $X^{2}=3.742, P=0.291$ & $315(75.4)$ & $X^{2}=4.372, P=0.224$ \\
\hline & Catholic & $5(4.8)$ & & $17(5.4)$ & & $22(5.3)$ & \\
\hline & Muslim & 11(10.6) & & $48(15.3)$ & & $59(14.1)$ & \\
\hline & Protestant & $7(6.7)$ & & $15(4.8)$ & & $22(5.3)$ & \\
\hline \multirow[t]{2}{*}{ Grade of students } & $1-4$ & $104(100)$ & - & $138(43.9)$ & $X^{2}=8.088, P=0.004$ & $242(57.9)$ & $X^{2}=14.555, P<0.000$ \\
\hline & $5-8$ & $0(0)$ & & $176(56.1)$ & & $176(42.1)$ & \\
\hline \multirow[t]{5}{*}{ Family size } & $<=3$ & $3(2.9)$ & $X^{2}=4.703, p=0.319$ & $71(22.6)$ & $X^{2}=32.357, P<0.000$ & $74(17.7)$ & $X^{2}=32.265, P<0.000$ \\
\hline & 4 & $22(21.2)$ & & $82(26.1)$ & & 104(24.9) & \\
\hline & 5 & $28(26.9)$ & & $59(18.8)$ & & $87(20.8)$ & \\
\hline & 6 & 19(18.3) & & $53(16.9)$ & & $72(17.2)$ & \\
\hline & $>=7$ & $32(30.8)$ & & 49(15.6) & & 81(19.4) & \\
\hline \multirow[t]{4}{*}{ Maternal Education } & No formal Education & $56(53.8)$ & $X^{2}=8.996, p=0.061$ & $54(17.2)$ & $X^{2}=2.238, P=0.692$ & $110(26.3)$ & $X^{2}=10.822, P=0.029$ \\
\hline & Grade 1-8 & $21(20.2)$ & & 113(36.0) & & 134(32.1) & \\
\hline & Grade $9-12$ & 18(17.3) & & $71(22.6)$ & & $89(21.3)$ & \\
\hline & Collage \& above & $9(8.7)$ & & $76(24.2)$ & & $85(20.3)$ & \\
\hline
\end{tabular}


Table 2 Hygiene and safety-related characteristics of mentally disabled and non-disabled primary school students' in Bahir Dar City, Amhara Regional State, Ethiopia, $2018(n=418)$

\begin{tabular}{|c|c|c|c|c|c|c|c|}
\hline \multicolumn{2}{|l|}{ Characteristics } & \multicolumn{6}{|c|}{ Students with disability status } \\
\hline & & \multicolumn{2}{|c|}{ Disabled students $(n=104)$} & \multicolumn{2}{|c|}{$\begin{array}{l}\text { Non-Disabled Students }(n= \\
314)\end{array}$} & \multicolumn{2}{|c|}{ Total $(n=418)$} \\
\hline Variables & Category & Freq (\%) & $x^{2}, p$ & Freq (\%) & $x^{2}, p$ & Freq (\%) & $x^{2}, p$ \\
\hline \multirow[t]{2}{*}{ Hand washing Habit } & water \& soap & $37(35.6)$ & $x^{2}=8.351, p=0.004$ & 144(45.9) & $X^{2}=30.930, P<0.000$ & $181(43.3)$ & $X^{2}=41.351, P<0.000$ \\
\hline & water only & $67(64.4)$ & & $170(54.1)$ & & $237(56.7)$ & \\
\hline \multirow[t]{2}{*}{ Hand washing before food } & Always & $49(47.1)$ & $x^{2}=7.265, p=0.007$ & 293(93.3) & $X^{2}=1.187, P=0.276$ & $342(81.8)$ & $X^{2}=14.269, P<0.000$ \\
\hline & Sometimes & $55(52.9)$ & & $21(6.7)$ & & $76(18.2)$ & \\
\hline \multirow[t]{3}{*}{ Hand washing after latrine } & Always & $40(38.5)$ & $X^{2}=5.365, p=0.068$ & $113(36.0)$ & $X^{2}=19.956, P<0.000$ & 153(36.6) & $X^{2}=22.789, P<0.000$ \\
\hline & Sometimes & $41(39.4)$ & & $132(42.0)$ & & 173(41.4) & \\
\hline & Never & $23(22.1)$ & & $69(22.0)$ & & $92(22.0)$ & \\
\hline \multirow[t]{4}{*}{ Fingernail cutting } & $>=2$ times per week & $25(24.0)$ & $X^{2}=2981, p=0.225$ & $102(32.5)$ & $X^{2}=26.686, P<0.000$ & $127(30.4)$ & $X^{2}=18.719, P<0.000$ \\
\hline & One time per week & $46(44.2)$ & & $77(24.5)$ & & $123(29.4)$ & \\
\hline & $<=1$ time per 2 weeks & $33(31.7)$ & & $96(30.6)$ & & 129(30.9) & \\
\hline & No need to cut & - & & $39(12.4)$ & & 39(9.3) & \\
\hline \multirow[t]{2}{*}{ Fingernail cleanliness } & Clean & $46(44.2)$ & $X^{2}=13.139, p<0.000$ & $238(75.8)$ & $X^{2}=34.012, P<0.000$ & $284(67.9)$ & $X^{2}=53.540, P<0.000$ \\
\hline & Not clean & $58(55.8)$ & & $76(24.2)$ & & 134(32.1) & \\
\hline \multirow[t]{3}{*}{ Shoes wearing at home } & Always & $32(30.8)$ & $X^{2}=12.541, p=0.002$ & $97(30.9)$ & $X^{2}=6.362, P=0.042$ & 129(30.9) & $X^{2}=12.685, P=0.002$ \\
\hline & Sometimes & $37(35.6)$ & & $142(45.2)$ & & 179(42.8) & \\
\hline & Never & $35(33.7)$ & & $75(23.9)$ & & $110(26.3)$ & \\
\hline \multirow[t]{2}{*}{ Shoe wearing at school } & Always & $83(79.8)$ & $X^{2}=4.059, p=0.044$ & $314(100)$ & - & $397(95.0)$ & $X^{2}=8.705, P=0.003$ \\
\hline & Sometimes & $21(20.2)$ & & $0(0)$ & & $21(5.0)$ & \\
\hline \multirow[t]{4}{*}{ Shower taking } & $<=1$ time per week & $77(74.0)$ & $X^{2}=1.946, p=0.584$ & 108(34.4) & $X^{2}=10.890, P=0.004$ & $185(44.3)$ & $X^{2}=17.756, P<0.000$ \\
\hline & 2 times per week & $13(12.5)$ & & $153(48.7)$ & & 166(39.7) & \\
\hline & $>=3$ times per week & $8(7.7)$ & & $53(16.9)$ & & $61(14.6)$ & \\
\hline & Never & 6(5.8) & & $0(0)$ & & $6(1.4)$ & \\
\hline
\end{tabular}

NB. Freq $=$ Frequency $\%=$ percent

\section{Distribution of IPIs based on mental disability status} Prevalence of single, double and triple parasitic infection was $38.50 \%(n=161), 25(5.90 \%)$ and $2(0.50 \%)$, respectively. Ten intestinal parasites were identified from both study groups. Among these single intestinal parasitic infections, E. histolytica/dispar was the most prevalent $12.00 \%(n=50)$ followed by $A$. lumbricoides 7.90\% $(n=33)$, Hookworm 6.20\% $(n=26)$ and G. lamblia $4.80 \%(n=20)$ [Table 5].

Factors associated with IPIs among mentally disabled and non-disabled primary school students

In multivariable logistic regression, hand washing habit, fingernail cleanliness, health checkup, family size, the source of cooking and sanitation water, sex of students and grade of students have had a statistically significant association with prevalence of intestinal parasitic infections among primary school students at $P$-value less than 0.05 .
Those students who had washed their hands using only water were 2.48 times more likely to develop an intestinal parasitic infection as compared with students who wash his/her hands with water and soap (AOR = $2.48 ; 1.45,4.12$ ).

Unclean fingernail was predictors' factors for intestinal parasitic infections for non-disabled students as well as for mentally disabled students. Students who have unclean fingernails were nearly 2.42 times more likely to infected by intestinal parasites than the counterpart (AOR $=2.42 ; 1.40,4.17)$.

Water sources were the strongest determinant factor for IPI on non-disabled students. Students whose household cooking and sanitation water source from both (well and tape) were 4.40 times more likely to develop IPI than students whose source of water from tape water only $(\mathrm{AOR}=4.40 ; 2.32,8.36)$.

Those students who had not any medical checkups previously were 1.87 times more likely to be infected with intestinal parasites than mentally disabled students 
Table 3 hygiene and safety-related characteristics of mentally disabled and non-disabled primary school students in Bahir Dar City, Amhara Regional State, Ethiopia, $2018(n=418)$

\begin{tabular}{|c|c|c|c|c|c|c|c|}
\hline \multicolumn{2}{|l|}{ Characteristics } & \multicolumn{6}{|c|}{ Students with disability status } \\
\hline & & \multicolumn{2}{|c|}{ Disabled Students $(\mathrm{n}=104)$} & \multicolumn{2}{|c|}{$\begin{array}{l}\text { Non-disabled students }(n= \\
314)\end{array}$} & \multicolumn{2}{|c|}{ Total $(n=418)$} \\
\hline Variables & Category & $\begin{array}{l}\text { Freq } \\
(\%)\end{array}$ & $x^{2}, p$ & $\overline{\text { Freq }(\%)}$ & $x^{2}, p$ & Freq $(\%)$ & $x^{2}, p$ \\
\hline \multirow[t]{4}{*}{ Water contact activity } & 1 time/week & $10(9.6)$ & \multirow{4}{*}{$\begin{array}{l}X^{2}=2.846, p= \\
0.416\end{array}$} & $54(17.2)$ & \multirow[t]{4}{*}{$X^{2}=7.002, P=0.072$} & $64(15.3)$ & \multirow[t]{4}{*}{$X^{2}=7.596, P=0.055$} \\
\hline & $\begin{array}{l}2-3 \text { times/ } \\
\text { week }\end{array}$ & $9(8.7)$ & & $42(13.4)$ & & $51(12.2)$ & \\
\hline & $\begin{array}{l}>3 \text { times/ } \\
\text { week }\end{array}$ & 2(1.9) & & $48(15.3)$ & & $50(12.0)$ & \\
\hline & Never & $83(79.8)$ & & $170(54.1)$ & & $253(60.5)$ & \\
\hline \multirow[t]{3}{*}{ Latrine utilization } & Always & $50(48.1)$ & \multirow{3}{*}{$\begin{array}{l}X^{2}=0.879, p= \\
0.644\end{array}$} & $314(100)$ & \multirow[t]{3}{*}{-} & $364(87.1)$ & \multirow[t]{3}{*}{$X^{2}=6.525, P=0.038$} \\
\hline & Sometimes & $41(39.4)$ & & $0(0)$ & & $41(9.8)$ & \\
\hline & Never & $13(12.5)$ & & $0(0)$ & & $13(3.1)$ & \\
\hline \multirow[t]{3}{*}{ Type of latrine at home } & Dry latrine & $35(33.7)$ & \multirow{3}{*}{$\begin{array}{l}X^{2}=9.044, p= \\
0.011\end{array}$} & 30(9.6) & \multirow[t]{3}{*}{$X^{2}=4.118, P=0.128$} & 65(15.6) & \multirow{3}{*}{$\begin{array}{l}X^{2}=13.618, P= \\
0.001\end{array}$} \\
\hline & Flush latrine & $25(24.0)$ & & $108(34.4)$ & & 133(31.8) & \\
\hline & Pour-flush & $44(42.3)$ & & $176(56.1)$ & & $220(52.6)$ & \\
\hline \multirow[t]{2}{*}{ Hand washing facility } & No & $55(52.9)$ & \multirow{2}{*}{$\begin{array}{l}x^{2}=2.268, p= \\
0.132\end{array}$} & $98(31.2)$ & \multirow[t]{2}{*}{$X^{2}=5.813, P=0.016$} & 153(36.6) & \multirow{2}{*}{$\begin{array}{l}X^{2}=10.915, P= \\
0.001\end{array}$} \\
\hline & Yes & $49(47.1)$ & & $216(68.8)$ & & $265(63.4)$ & \\
\hline \multirow[t]{3}{*}{ Defecation modality } & Using latrine & $45(43.3)$ & \multirow{3}{*}{$\begin{array}{l}X^{2}=6.816, p= \\
0.033\end{array}$} & $314(100)$ & \multirow[t]{3}{*}{-} & $359(85.9)$ & \multirow{3}{*}{$\begin{array}{l}X^{2}=14.470, P= \\
0.001\end{array}$} \\
\hline & Open field & $41(39.4)$ & & $0(0)$ & & $41(9.8)$ & \\
\hline & Using popo & 18(17.3) & & $0(0)$ & & $18(4.3)$ & \\
\hline \multirow[t]{2}{*}{ Previous health checks } & No & $68(65.4)$ & \multirow{2}{*}{$\begin{array}{l}X^{2}=12.279, p< \\
000\end{array}$} & $165(52.5)$ & \multirow{2}{*}{$\begin{array}{l}X^{2}=13.871, P< \\
0.000\end{array}$} & 233(55.7) & \multirow{2}{*}{$\begin{array}{l}X^{2}=26.910, P< \\
0.000\end{array}$} \\
\hline & Yes & $36(34.6)$ & & $149(47.5)$ & & 185(44.3) & \\
\hline \multirow{4}{*}{$\begin{array}{l}\text { When do you have a health } \\
\text { checkups }\end{array}$} & $<1$ year & $28(26.9)$ & \multirow{4}{*}{$\begin{array}{l}x^{2}=6.907, p= \\
0.009\end{array}$} & 97(30.9) & \multirow{4}{*}{$\begin{array}{l}X 2=14.501, P= \\
0.002\end{array}$} & 125(29.9) & $X 2=27.154, P<$ \\
\hline & 1 year-2 year & $5(4.8)$ & & 28(8.9) & & $33(7.9)$ & \\
\hline & $>2$ years & $3(2.9)$ & & $24(7.6)$ & & $27(6.5)$ & \\
\hline & Never & $68(65.4)$ & & $165(52.5)$ & & 233(55.7) & \\
\hline Ways of transportation & Car/taxi & $26(25.0)$ & $x^{2}=1.499, p=$ & $73(23.2)$ & $X^{2}=26.124, P<$ & $99(23.7)$ & $X^{2}=22.265, P<$ \\
\hline & Foot & $24(23.1)$ & $0.4 / 3$ & $85(27.1)$ & 0.000 & 109(26.1) & \\
\hline & Both & 54(51.9) & & 156(49.7) & & $210(50.2)$ & \\
\hline
\end{tabular}

NB. Freq $=$ Frequency, $\%=$ percent

who had medical checkups previously $(\mathrm{AOR}=1.87 ; 95 \%$ CI: 1.16,3.02).

The probability of being infected with intestinal parasites for primary school students from a family size of 7 and above was higher than those from a family size of less than or equal to 3 (AOR:2.63; 95\%CI:1.209-5.72).

Students in a grade of 1-4 were more likely to be infected with intestinal parasitic infections than those in a grade of 5-8 (AOR: 2.20; 95\%CI: 1.37-3.54).

Male primary school students were 1.642 times more likely to be positive for IPIs than females (AOR: 1.64; 95\%CI: 1.03-2.63) [Tables 6 and 7].

\section{Discussion}

Epidemiological investigations on the prevalence of intestinal parasitic infection and associated risk factors in primary school students are necessary to design appropriate intervention strategies. This study determined the prevalence of intestinal parasitic infections and associated factors among mentally disabled and non-disabled primary school students in Bahir Dar, Amhara Regional State, Ethiopia. The study revealed that 56.70\% (95\% CI: $47.20-66.20$ ) of mentally disabled and $41.10 \%$ (95\% CI: 35.70-46.50) non-disabled primary school students were infected with intestinal parasitic infections. Prevalence of intestinal parasitic infections was higher among mentally disabled students than non-disabled students with point estimate for the difference is $15.60 \%$ (95\% CI: $0.05,0.27)$. This suggested that mental disability can directly influence hygiene and safety habits and, consequently, favor the acquisition of intestinal parasitic infections. 
Table 4 Food and drink establishments' related characteristics of mentally disabled and non-disabled primary school students in Bahir Dar City, Amhara Regional State, Ethiopia, $2018(n=418)$

\begin{tabular}{|c|c|c|c|c|c|c|c|}
\hline \multicolumn{2}{|l|}{ Characteristics } & \multicolumn{6}{|c|}{ Students with disability status } \\
\hline & & \multicolumn{2}{|c|}{ Disabled students $(n=104)$} & \multicolumn{2}{|c|}{ Non-disabled students $(n=314)$} & \multicolumn{2}{|c|}{ Total $(\mathrm{n}=418)$} \\
\hline Variables & Category & $\begin{array}{l}\text { Freq } \\
(\%)\end{array}$ & $x^{2}, p$ & Freq (\%) & $x^{2}, p$ & Freq (\%) & $x^{2}, p$ \\
\hline \multirow[t]{4}{*}{ Eating raw meat } & $\begin{array}{l}<=1 \text { time per } \\
\text { week }\end{array}$ & $28(26.9)$ & \multirow[t]{4}{*}{$X^{2}=0.254, p=0.881$} & $61(19.4)$ & \multirow[t]{4}{*}{$X^{2}=4.624, P=0.202$} & $89(21.3)$ & \multirow[t]{4}{*}{$X^{2}=3.880, P=0.275$} \\
\hline & 2-3 times per week & $12(11.5)$ & & $50(15.9)$ & & $62(14.8)$ & \\
\hline & $>3$ times per week & $0(0)$ & & $15(4.8)$ & & 15(3.6) & \\
\hline & Never & $64(61.5)$ & & 188(59.9) & & $252(60.3)$ & \\
\hline \multirow[t]{4}{*}{ Eating raw vegetables } & $\begin{array}{l}<=1 \text { time per } \\
\text { week }\end{array}$ & $34(32.7)$ & \multirow[t]{4}{*}{$\begin{array}{l}X^{2}=4.922, p= \\
0.178\end{array}$} & $49(15.6)$ & \multirow[t]{4}{*}{$\begin{array}{l}X^{2}=11.754, P= \\
0.008\end{array}$} & $83(19.9)$ & \multirow[t]{4}{*}{$X^{2}=4.274, P=0.233$} \\
\hline & 2-3 times per week & $9(8.7)$ & & $121(38.5)$ & & 130(31.1) & \\
\hline & $>3$ times per week & $9(8.7)$ & & $59(18.8)$ & & $68(16.3)$ & \\
\hline & Never & $52(50.0)$ & & $85(27.1)$ & & 137(32.8) & \\
\hline \multirow[t]{4}{*}{$\begin{array}{l}\text { Eating/consuming/street } \\
\text { food }\end{array}$} & $\begin{array}{l}<=1 \text { time per } \\
\text { week }\end{array}$ & $61(58.7)$ & \multirow[t]{4}{*}{$\begin{array}{l}X^{2}=4.690, p= \\
0.196\end{array}$} & $85(27.1)$ & \multirow[t]{4}{*}{$X^{2}=5.255, P=0.154$} & $146(34.9)$ & \multirow[t]{4}{*}{$X^{2}=4.113, P=0.250$} \\
\hline & 2-3 times per week & 16(15.4) & & $94(29.9)$ & & $110(26.3)$ & \\
\hline & $>3$ times per week & $8(7.7)$ & & $78(24.8)$ & & $86(20.6)$ & \\
\hline & Never & 19(18.3) & & $57(18.2)$ & & $76(18.2)$ & \\
\hline \multirow[t]{2}{*}{ Cooking \&sanitation water } & Tape water & $94(90.4)$ & \multirow{2}{*}{$\begin{array}{l}X^{2}=0.794, p= \\
0.373\end{array}$} & 249(79.3) & \multirow{2}{*}{$\begin{array}{l}X^{2}=36.351, P< \\
0.000\end{array}$} & $343(82.1)$ & \multirow{2}{*}{$\begin{array}{l}X^{2}=29.699, P< \\
0.000\end{array}$} \\
\hline & Tape \& well water & 10(9.6) & & $65(20.7)$ & & $75(17.9)$ & \\
\hline
\end{tabular}

NB. Freq $=$ Frequency, $\%=$ percent

The prevalence of IPI among mentally disabled students in this study $56.7 \%$ was higher than else studies conducted in Rasht, Northern Iran (5.15\%) [18], Brazil (8.30\%) [19], Tanzania (12.45\%) [20] and Egypt (43.50\%) [14]. Because it might be due to poor shoe wearing practices, poor hand washing habits, open defecation practices, poor personal and environmental hygiene and limited health checkup.
In another way, this study was lower than the study conducted in Iran among elderly and mentally retarded residence $(78.70 \%)$ [21]. This might be due to the fact that the study participants in the latter study is carried out among elderly individuals in the community while the current study done on mentally disabled students at school which indicate most of the mentally disabled

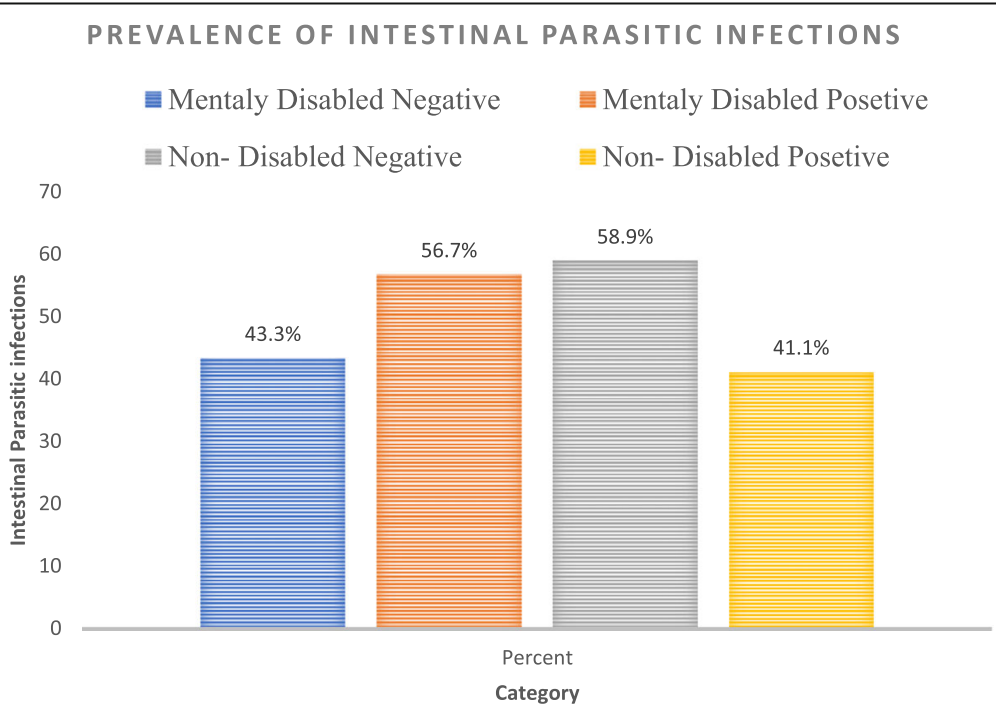

Fig. 1 Prevalence of IPIs with respect to disability status in Bahir Dar City, Amhara Regional State, Ethiopia, $2018(n=418)$ 
Table 5 Distribution of intestinal parasitic infections among mentally disabled and non-disabled primary school students in Bahir Dar, Amhara Regional State, Ethiopia, 2018

\begin{tabular}{|c|c|c|c|c|}
\hline \multicolumn{2}{|c|}{ Intestinal parasitic infections } & \multirow{2}{*}{$\begin{array}{l}\text { Mentally disabled }(n=104) \\
\text { No. of infections }(\%)\end{array}$} & \multirow{2}{*}{$\begin{array}{l}\text { Non-disabled }(n=314) \\
\text { No. of infections (\%) }\end{array}$} & \multirow{2}{*}{$\begin{array}{l}\text { Total }(n=418) \\
\text { No. of infections }(\%,\end{array}$} \\
\hline & & & & \\
\hline \multirow[t]{8}{*}{ Helminths } & A. lumbricoides & 10(9.6) & $23(7.3)$ & $33(7.9)$ \\
\hline & Hookworm & $7(6.7)$ & $19(6.1)$ & $26(6.2)$ \\
\hline & S. Stercoralis & $4(3.8)$ & $1(0.3)$ & $5(1.2)$ \\
\hline & Taenia species & $1(1.0)$ & $5(1.6)$ & $6(1.4)$ \\
\hline & T. trichiura & 0 & $3(1.0)$ & $3(0.7)$ \\
\hline & H. nana & $4(3.8)$ & $4(1.3)$ & $8(1.9)$ \\
\hline & E. vermicularis & $4(3.8)$ & 0 & $4(1.0)$ \\
\hline & S. mansoni & $1(1.0)$ & $5(1.6)$ & $6(1.4)$ \\
\hline \multirow[t]{2}{*}{ Protozoa } & E. histolytica & $15(14.4)$ & $35(11.1)$ & $50(12.0)$ \\
\hline & G. lamblia & $6(5.8)$ & $14(4.5)$ & $20(4.8)$ \\
\hline \multirow[t]{5}{*}{ Double infections } & A. lumbricoides + E. histolytica & $1(1.0)$ & $10(3.2)$ & $11(2.6)$ \\
\hline & A. lumbricoides + Hookworm & 2(1.9) & $3(1.0)$ & $5(1.2)$ \\
\hline & A. lumbricoides + S. stercoralis & $1(1.0)$ & 0 & $1(0.2)$ \\
\hline & Hookworm + E. histolytica & 2(1.9) & $5(1.6)$ & $7(1.7)$ \\
\hline & T. trichiura + S. mansoni & $1(1.0)$ & 0 & $1(0.2)$ \\
\hline Triple infections & A. lumbricoides + Hookworm + E. histolytica & 0 & $2(0.6)$ & $2(0.5)$ \\
\hline Overall infections & & $59(56.7)$ & 129(41.1) & 188(45) \\
\hline
\end{tabular}

students at school may have better follow-up on their personal hygiene and sanitation through teachers and guardian than elderly mentally disabled individuals in the community which directly affect the prevalence of intestinal parasitic infections.

Similarly, the prevalence of IPI among non-disabled students in this study ( $P=41.10,95 \%$ CI: $35-46.20 \%$ ) was similar with a previous study conducted in Turkey 44.6\% [22], Southern Ethiopia 39.9\% [10] and in Amhara Region, Tillili (44.2\%) [23]. However, this finding was lower than most studies done worldwide (Peru 47\% [24], Argentina 78.30\% [25] and Yemen 54.80\% [26]) in Africa (South Africa 64.80\% [8], Rwanda 50.50\% [9], Sao Tome 64.70\% [27] and Tanzania 48.70\% [28]) and in Ethiopia, (Chencha Town, 81\% [11], Jimma, 48.40\% [29], Mizan Aman Town, 76.70\% [30], and Bahir Dar 65.50\% [16]). The most possible reasons were an improvement in proper and protected drinking water supply, better shoe wearing practices at school and improvement on personal and environmental hygiene and sanitation.

In addition to determining the PIPIs, this study also assesses various risk factors of intestinal parasitic infections among mentally disabled and non-disabled students.

The study revealed that unclean fingernails/having dirty materials in fingernails made students be more infected with intestinal parasites $(\mathrm{AOR}=2.42 ; 1.40,4.17)$. A Comparable association of intestinal parasitic infection with the availability of dirty material in fingernail were reported in different studies conducted in different period time and different parts of Ethiopia [16, 31-34]. This could be described by the availability of dirty materials in fingernails may lead to direct feco-oral transmission of intestinal parasite or it uses as habitat to proceed the life cycle of soil-transmitted intestinal parasites.

The previous medical checkup was one of the risk factors for intestinal parasitic infections. Students who had not any medical checkups previously were more likely to be infected with intestinal parasites than students who had medical checkups previously ( $\mathrm{AOR}=1.87 ; 1.16,3.02)$, Which is in line with a study conducted in Aksum, Ethiopia [35]. This might indicate mentally disabled students who visit health institution with his/her parents or guardian had a tendency to decrease intestinal parasitic infections through increasing his/her health, safety, and personal hygiene.

The risk of acquiring infections among students who used water only during hand washing was 2.48 times higher than among those who used water and soap during hand washing $(\mathrm{AOR}=2.48 ; 1.49,4.12)$ which is congruent with earlier studies conducted in Ethiopia [36], [29]. This might due to using water only didn't sufficient to keep our safety and hygiene, so students must use water with soap to remove infective stage of the parasite or dirty materials from those hand.

Students whose household cooking and sanitation water source from both (well and tape) were 4.40 times more likely to develop IPI than students whose source of water from tape water only $(\mathrm{AOR}=4.40 ; 2.32,8.36)$, this 
Table 6 Bi-variable and Multivariable analysis of IPI among mentally disabled and non-disabled students in Bahir Dar City, Amhara Regional State, Ethiopia

\begin{tabular}{|c|c|c|c|c|c|}
\hline \multirow[t]{2}{*}{ Variables } & \multirow[t]{2}{*}{ Category } & \multicolumn{2}{|l|}{$\underline{\mathrm{IPI}}$} & \multirow[t]{2}{*}{ COR $(95 \% \mathrm{Cl})$} & \multirow[t]{2}{*}{ AOR $(95 \% \mathrm{Cl})$} \\
\hline & & Yes, N (\%) & No, N (\%) & & \\
\hline \multirow[t]{2}{*}{ Mental disability } & Non-disabled & 129(30.9) & $185(44.2)$ & 1 & - \\
\hline & Disabled & $59(14.1)$ & $45(10.8)$ & $1.880(1.201,2.944) * *$ & - \\
\hline Age & - & $188(45)$ & $230(55)$ & $0.919(0.865,0.976) * *$ & - \\
\hline \multirow[t]{2}{*}{ Sex of students } & Male & 104(24.9) & $98(23.4)$ & $1.668(1.131,2.459) *$ & $1.642(1.026,2.627) *$ \\
\hline & Female & $84(20.1)$ & 132(31.6) & 1 & 1 \\
\hline \multirow[t]{2}{*}{ Grade of Students } & 1st cycle [1-4] & 128(30.6) & $114(27.3)$ & $2.171(1.454,3.242) * * *$ & $2.272(1.408,3.666) * *$ \\
\hline & $2^{\text {ed }}$ cycle [5-8] & $60(14.4)$ & $116(27.8)$ & 1 & \\
\hline \multirow[t]{5}{*}{ Family size } & $<=3$ & $22(5.3)$ & $52(12.4)$ & 1 & 1 \\
\hline & 4 & $34(8.1)$ & $70(16.7)$ & $1.148(0.602,2.189)$ & $1.015(0.484,2.131)$ \\
\hline & 5 & $39(9.3)$ & $48(11.5)$ & $1.920(0.999,3.691)$ & $1.211(0.565,2.595)$ \\
\hline & 6 & $38(9.1)$ & $34(8.1)$ & $2.642(1.338,5.215) *$ & $1.747(0.794,3.841)$ \\
\hline & $>=7$ & $55(13.2)$ & $26(6.2)$ & $5.000(2.526,9.896) * * *$ & $2.736(1.249,5.994) *$ \\
\hline \multirow[t]{4}{*}{ Maternal Education } & No formal education & $61(14.6)$ & $49(11.7)$ & $2.282(1.275,4.086) * *$ & - \\
\hline & $1-8$ & $64(15.3)$ & $70(16.7)$ & $1.676(0.958,2.932)$ & - \\
\hline & $9-12$ & $33(7.9)$ & $56(13.4)$ & $1.080(0.582,2.006)$ & - \\
\hline & College \& above & $30(7.2)$ & $55(13.2)$ & 1 & - \\
\hline \multirow[t]{2}{*}{ Hand washing Habit } & Water \& soup & 49(11.7) & 132(31.6) & 1 & \\
\hline & Water only & 139(33.3) & $98(23.4)$ & $3.821(2.517,5.801) * * *$ & $2.476(1.489,4.119) * * *$ \\
\hline \multirow[t]{2}{*}{ Hand washing before food } & Always & 139(33.3) & 203(48.6) & 1 & - \\
\hline & Sometimes & $49(11.7)$ & $27(6.5)$ & $2.650(1.581,4.444)^{* * *}$ & - \\
\hline \multirow[t]{3}{*}{ Hand washing habit after latrine } & Always & 48(11.5) & $105(25.1)$ & 1 & - \\
\hline & Sometimes & 83(19.9) & $90(21.5)$ & $2.017(1.282,3.175) * *$ & - \\
\hline & Never & 57(13.6) & $35(8.4)$ & $3.562(2.072,6.126) * * *$ & - \\
\hline \multirow[t]{4}{*}{ Fingernail cutting } & $>=2$ times per week & $39(9.3)$ & $88(21.1)$ & 1 & - \\
\hline & 1 time per week & $55(13.2)$ & $68(16.3)$ & $1.825(1.087,3.064) *$ & - \\
\hline & 1 time per 2 weeks & $73(17.5)$ & $56(13.4)$ & $2.941(1.761,4.914) * * *$ & - \\
\hline & No need of to cut & $21(5.0)$ & $18(4.3)$ & $2.632(1.264,5.484) *$ & - \\
\hline \multirow[t]{2}{*}{ Fingernail cleanliness } & Clean & $93(22.2)$ & $191(45.7)$ & 1 & \\
\hline & Not clean & $95(22.7)$ & $39(9.3)$ & $5.003(3.198,7.827) * * *$ & $2.416(1.400,4.168) * *$ \\
\hline
\end{tabular}

Note: * statistically significant at $P<0.05,{ }^{* *}<0.01,{ }^{* * *}<0.001$

result also inconsistent with $[16,29,37]$. The possible explanations of the association of water source and increase parasitic infection might be due to an increased chance of contamination of well water by infective stages of different intestinal parasites.

The probability of being infected by intestinal parasites was increased by about 2.74 folds among students belonging in a family size of $>=7$ as compared with students belonging in $<=3$ family size $(\mathrm{AOR}=2.74 ; 1.25$, 5.99). Other studies in agreement with this study conducted in Ethiopia were [18] and [33]. This association might be due to the fact that personal hygiene, environmental sanitation, and other nutritional related problems. As a family size increase, there might be a problem of overcrowding, under-nutrition, poor sanitation, and personal hygiene which enhance intestinal parasitic infection susceptibility.

Furthermore, males were 1.64 times high risk for intestinal parasitic infections than females $(\mathrm{AOR}=1.64$; $1.03,2.63)$. This is comparable to a study conducted in Southwest Ethiopia [29]. Male students usually play outdoors and participate in outdoor activities compared to females, which may enhance the risks of IPI. However, this finding contradicts with another study conducted in Ethiopia [30].

Lastly, there was a significant association between the educational level of students and the rate of intestinal parasitic infections. Students in the first cycle were 2.27 
Table 7 Bi-variable and Multivariable analysis of IPI among mentally disabled and non-disabled students in Bahir Dar City, Amhara Regional State, Ethiopia

\begin{tabular}{|c|c|c|c|c|c|}
\hline \multirow[t]{2}{*}{ Variables } & \multirow[t]{2}{*}{ Category } & \multicolumn{2}{|l|}{$\underline{I P I}$} & \multirow[t]{2}{*}{ COR $(95 \% \mathrm{Cl})$} & \multirow[t]{2}{*}{ AOR $(95 \% \mathrm{Cl})$} \\
\hline & & Yes, N (\%) & No, N (\%) & & \\
\hline \multirow[t]{3}{*}{ Shoe wearing habit at home } & Always & $48(11.5)$ & $81(19.4)$ & 1 & - \\
\hline & Sometimes & $75(17.9)$ & 104(24.9) & $1.217(0.765,1.936)$ & - \\
\hline & Never & 65(15.6) & $45(10.8)$ & $2.437(1.447,4.106) * *$ & - \\
\hline \multirow[t]{2}{*}{ Shoe wearing habit at school } & Always & $172(41.1)$ & $225(53.8)$ & 1 & - \\
\hline & Sometimes & $16(3.8)$ & $5(1.2)$ & $4.186(1.504,11.651) * *$ & - \\
\hline \multirow[t]{3}{*}{ Frequency of shower } & $<=1$ times per week & $106(24.5)$ & $85(20.3)$ & $2.060(1.141,3.721) *$ & - \\
\hline & 2 times per week & $59(14.1)$ & 107(25.6) & $0.911(0.496,1.673)$ & - \\
\hline & $>=3$ times per week & $23(5.5)$ & $38(9.1)$ & 1 & - \\
\hline \multirow[t]{4}{*}{ Water contact activity } & 1 time per week & $28(6.7)$ & $36(8.6)$ & $0.809(0.466,1.405)$ & - \\
\hline & 2-3 times per week & $22(5.3)$ & $29(6.9)$ & $0.789(0.430,1.447)$ & - \\
\hline & $>3$ times per week & $14(3.3)$ & $36(8.6)$ & $0.405(0.208,0.786) * *$ & - \\
\hline & Never & $124(29.7)$ & $129(30.9)$ & 1 & - \\
\hline \multirow[t]{3}{*}{ Latrine utilization } & Always & 155(37.1) & 209(50) & 1 & - \\
\hline & Sometimes & $25(6)$ & $16(3.8)$ & $2.107(1.088,4.080) *$ & - \\
\hline & Never & $8(1.9)$ & $5(1.2)$ & $2.157(0.692,6.722)$ & - \\
\hline \multirow[t]{3}{*}{ Type of latrine used at home } & Dry/pit latrine & $41(9.8)$ & $24(5.7)$ & $2.050(1.160,3.623) *$ & - \\
\hline & Flash latrine & $47(11.2)$ & $86(20.6)$ & $0.656(0.421,1.022)$ & - \\
\hline & pour flash latrine & $100(23.9)$ & $120(28.7)$ & 1 & - \\
\hline \multirow[t]{2}{*}{ Hand washing facility around the latrine } & No & $85(20.3)$ & $68(16.3)$ & $1.966(1.313,2.943) * *$ & - \\
\hline & Yes & 103(24.6) & 162(38.8) & 1 & \\
\hline \multirow[t]{3}{*}{ Ways of defecation } & Using latrine & 148(35.4) & $211(50.5)$ & 1 & - \\
\hline & Open field & $28(6.7)$ & $13(3.1)$ & $3.071(1.539,6.125) * *$ & - \\
\hline & Using popo & $12(2.9)$ & $6(1.4)$ & $2.851(1.047,7.768) *$ & - \\
\hline \multirow[t]{2}{*}{ Health checkup } & No & $131(31.3)$ & $102(24.4)$ & $2.884(1.923,4.326) * * *$ & $1.869(1.156,3.023) *$ \\
\hline & Yes & $57(13.6)$ & 128(30.6) & 1 & 1 \\
\hline \multirow[t]{3}{*}{ Modes of transportation } & Car & $32(7.7)$ & $67(16.0)$ & 1 & - \\
\hline & Foot & $69(16.5)$ & $40(9.6)$ & $3.612(2.035,6.410) * * *$ & - \\
\hline & Both & $87(20.8)$ & $123(29.4)$ & $1.481(0.896,2.448)$ & - \\
\hline \multirow[t]{2}{*}{ Source of cooking \& sanitation water } & Tap water & 133(31.8) & $210(50.2)$ & 1 & \\
\hline & well \& Tape & $55(13.2)$ & $20(4.8)$ & $4.342(2.490,7.571) * * *$ & $4.404(2.319,8.364) * *$ \\
\hline
\end{tabular}

Note: * statistically significant at $P<0.05,{ }^{* *}<0.01,{ }^{* * *}<0.001$

times more likely to be infected with intestinal parasitic infections than those in the grade level of the second cycle $(\mathrm{AOR}=2.27 ; 1.41,3.67)$. This was in line with other studies $[23,36]$. This finding might be due to lack of regular health education program in the school which can decrease their awareness in the prevention and control mechanisms.

\section{Strength and limitation of the study Strength of the study}

Both wet mount and formol-ether concentration techniques were used to examine the presence of intestinal parasite from study participants stool sample which used to increase the validity of the measurement of the dependent variable.

The overall ability of direct wet mount microscopy to correctly diagnose intestinal helminths (Test Efficacy $=(\mathrm{TP}+\mathrm{TN}) /(\mathrm{TN}+\mathrm{TP}+\mathrm{FN}+\mathrm{FP}))$ was $94 \%$, While, its sensitivity $(\mathrm{S}=\mathrm{TP} / \mathrm{TP}+\mathrm{FN})$ and negative predictive value $(\mathrm{PPV}=\mathrm{TP} /(\mathrm{TP} / \mathrm{FP})$ were 76 and $92.7 \%$ respectively by taking formol-ether concentration techniques as gold standard method [38].

\section{Limitation of the study}

First, in this study, an interview was held with each student for non-disabled students while parents/guardian/ 
were asked about their mentally disabled children using a questionnaire pertaining to factors associated with intestinal parasitic infections.

Second, as we only examined a single stool samples by both wet mount and concentrations technique of each study participant, we might underestimate the true prevalence of parasitic infections, due to cyclical nature of life cycle of intestinal parasites, to say negative stool sample at list three consecutive samples in three consecutive days must be examined from each study participants.

\section{Conclusion}

The present study concludes that the prevalence of intestinal parasitic infection among mentally disabled students was higher than the prevalence of intestinal parasitic infections among non-disabled primary school students. However, the prevalence of intestinal parasitic infection among non-disabled students was still high.

Among the different potential risk factors assessed in the study, fingernail cleanliness, previous health checkup, hand washing habits, cooking and sanitation source of water, family size, sex, and educational level were had a statistically significant association with intestinal parasitic infections among primary school students (Additional file 1).

\section{Additional file}

Additional file 1: Laboratory diagnosis of intestinal parasites. (DOCX 18 kb)

\section{Abbreviations}

AOR: Adjusted Odd Ratio; Cl: Confidence Interval; IP: Intestinal Parasites; IPI: Intestinal Parasitic Infection; PIPI: Prevalence of Intestinal Parasitic Infection; SPSS: Statistical Package for Social Science

\section{Acknowledgments}

Firstly, I give thanks to the Almighty God for strengthening me for doing this thesis.

I would like to acknowledge Bahir Dar University, College of Medicine and Health Science, teachers and other staff for giving me a chance to develop this thesis research.

Special thanks, to all study participants, parents/guardians' students, teachers of primary school students, laboratory personnel and data collectors who participated in this study, I thank you because without their participation; this study would not have been possible to conduct.

In addition, I would like to acknowledge Family guidance association of Ethiopia, Bahir Dar Model Sexual and Reproductive health clinic for giving permission to perform stool examination.

Lastly, I would like to acknowledge everybody that I have not mentioned above, your technical support and contribution were a blessing and God will bless you abundantly.

\section{Consent to publication}

Written assent form from parents/guardians of primary school students and consent from the students were obtained that the result of this research thesis would be included in publications.

\section{Authors' contributions}

AF was involved in the design, statistical analysis, and interpretation and manuscript drafting. $A A$ and $A B$ were participated in this thesis research by giving suggestions, comments, supports, encouragement, and contribution from the beginning of the research proposal throughout the thesis work.SM was involved in manuscript drafting, critical interpretation, and critical revision. All authors read and have given approval for this manuscript to be published.

Funding

No fund was obtained.

\section{Availability of data and materials}

The data can be accessed from the corresponding author through the following address fentahun143@gmail.com // agumlt@yahoo.com. The data will be accessed for research purpose and this is because, during the ethical clearance process, we agree with the Institutional review board of Bahir Dar University to keep the confidentiality of the data set.

\section{Ethics approval and consent to participate}

Ethical clearance for the proposed research was obtained from the research Ethical Review Board of Bahir Dar University College of Medicine and Health Sciences, School of Public Health. Letters of support were received from the university. Before the beginning of data collection, permission letter was obtained from Amhara National Regional Institute of Public Health and from each concerned body prior to the data collection period. The head of the school or directors of the school were informed about the purpose of the study that it will contribute for the health needs of the students, other people in the school, population in the study area and in the country at large. Before collecting the data, for mentally disabled students written consent from the parents/guardians, for non-disabled students age 7-12 years written consent from parents/guardians and assent from students and for students' age 13-17 assents from students only with parental/guardian permission were secured. From students, age $>17$ consent from students only secured. Names of the respondents did not use to ensure anonymity and confidentiality. All information obtained from the schools \& respondents were kept confidential. Individuals found to be positive for intestinal parasites were referred to the nearest health center for appropriate treatment and health education about personal hygiene, food hygiene and safety measures and about ways of IPIs transmission and prevention modalities were given to study participants

\section{Competing interests}

The authors declare that they have no competing interests.

\section{Author details}

${ }^{1}$ Family Guidance Association of Ethiopia, Bahir Dar Model Sexual and Reproductive Health Clinic, Bahir Dar, Ethiopia. ${ }^{2}$ School of Public Health, College of Medicine and Health Sciences, Bahir Dar University, Bahir Dar, Ethiopia. ${ }^{3}$ University of Gondar, and Health, Injibara, Ethiopia. ${ }^{4}$ Injibara Woreda Health Office, Injibara, Ethiopia.

Received: 23 March 2019 Accepted: 4 June 2019

Published online: 21 June 2019

\section{References}

1. Ali Y. Intestinal parasitic Infections among School-age Children In Mekaneselam Health Center. Borena: Addis Ababa University; 2016.

2. Chiodini PL, Moody AH, Manser DW, Jeffrey HC. Atlas of medical helminthology and protozoology. London: Churchill Livingstone Edinburgh; 2001.

3. Ouattara M, N'Guéssan NA, Yapi A, N'Goran EK. Prevalence and spatial distribution of Entamoeba histolytica/dispar and Giardia lamblia among schoolchildren in Agboville area (Côte d'Ivoire). PLoS Negl Trop Dis. 2010; 4(1):e574.

4. Haque R. Human intestinal parasites. J Health Popul Nutr. 2007;25(4):387.

5. Murray PR, Rosenthal KS, Pfaller MA. Medical Microbiology. 6th ed; 2008.

6. Organization WH. Partners for parasite control: geographical distribution and useful facts and stats, vol. 3. Geneva: World Health Organization; 2007

7. Hotez PJ, Fenwick A, Savioli L, Molyneux DH. Rescuing the bottom billion through control of neglected tropical diseases. Lancet. 2009; 373(9674):1570-5.

8. Nxasana N, Baba K, Bhat V, Vasaikar S. Prevalence of intestinal parasites in primary school children of Mthatha, eastern Cape Province, South Africa. Ann Med Health Sci Res. 2013;3(3):511-6. 
9. Niyizurugero E, Ndayanze JB, Bernard K. Prevalence of intestinal parasitic infections and associated risk factors among Kigali Institute of Education students in Kigali, Rwanda. Trop Biomed. 2013;30(4):718-26.

10. Wegayehu T, Tsalla T, Seifu B, Teklu T. Prevalence of intestinal parasitic infections among highland and lowland dwellers in Gamo area, South Ethiopia. BMC Public Health. 2013;13:151.

11. Abossie A, Seid M. Assessment of the prevalence of intestinal parasitosis and associated risk factors among primary school children in Chencha town, southern Ethiopia. BMC Public Health. 2014;14:166

12. WHO. World report on disability: WHO library cataloguing; 2011. Available from: https://www.who.int/disabilities/world_report/2011/report.pdf. Cited 2018 10/12

13. UN DoEaSA. Global Status Report on Disability and Development Prototype 2015.2015 Retrieved June 282018 from https://www.unicef.org/disabilities/ files/Factsheet_A5_Web_REVISED.pdf.

14. Shehata Al, Hassanein F. Intestinal parasitic infections among mentally handicapped individuals in Alexandria, Egypt. Annals Parasitol. 2015;61(4): 275-81

15. BCEO. Bahir Dar city educational office reports on number of primary school children. 2018.

16. Hailegebriel T. Prevalence of intestinal parasitic infections and associated risk factors among students at Dona Berber primary school, Bahir Dar, Ethiopia. BMC Infect Dis. 2017;17(1):362.

17. Cheesbrough M. District laboratory practice in tropical countries. PRESS CU, editor. Second Edition ed 2006.

18. Soleymani E, Davoodi L, Azami D. The prevalence of intestinal parasitic infections among the mentally retarded patients in Lamook Rehabilitation Center of Qaemshahr, Mazandaran Province, 2015. Tabari J Prev Med. 2016;2(1):1-5.

19. de Freitas JT, da Silva Matos J, Scarabeli SC, Fonseca ABM, da Silva Barbosa A, Bastos OMP, et al. Intestinal parasites in children with neurological disorders treated at a rehabilitation institution in Niterói, Rio de Janeiro, Brazil. Revista Patologia Tropical. 2013;46(2):171-84.

20. Nyundo AA, Munisi DZ, Gesase AP. Prevalence and correlates of intestinal parasites among patients admitted to Mirembe National Mental Health Hospital, Dodoma, Tanzania. J Parasitol Res. 2017;2017:5651717.

21. Rasti S, Arbabi M, Hooshyar H. High prevalence of Entamoeba histolytica and Enterobius vermicularis among elderly and mentally retarded residence in Golabchi center, Kashan, Iran 2006-2007. Jundishapur J Microbiol. 2012; 5(4):585-9.

22. Yentur Doni N, Gurses G, Simsek Z, Yildiz Zeyrek F. Prevalence and associated risk factors of intestinal parasites among children of farm workers in the southeastern Anatolian region of Turkey. Ann Agric Environ Med. 2015;22(3):438-42.

23. Abera A, Nibret E. Prevalence of gastrointestinal helminthic infections and associated risk factors among schoolchildren in Tilili town, Northwest Ethiopia. Asian Pac J Trop Med. 2014;7(7):525-30.

24. Cabada MM, Goodrich MR, Graham B, Villanueva-Meyer PG, Deichsel EL, Lopez M, et al. Prevalence of intestinal helminths, anemia, and malnutrition in Paucartambo, Peru. Rev Panam Salud Publica. 2015;37(2):69-75.

25. Dib J, Fernández-Zenoff M, Oquilla J, Lazarte S, González S. Prevalence of intestinal parasitic infection among children from a shanty town in Tucuman, Argentina. Trop Biomed. 2015;32(2):210-5.

26. Al-Mekhlafi AM, Abdul-Ghani R, Al-Eryani SM, Saif-Ali R, Mahdy MA. Schoolbased prevalence of intestinal parasitic infections and associated risk factors in rural communities of Sana'a, Yemen. Acta Trop. 2016;163:135-41.

27. Liao CW, Fu CJ, Kao CY, Lee YL, Chen PC, Chuang TW, et al. Prevalence of intestinal parasitic infections among school children in capital areas of the Democratic Republic of Sao Tome and Principe, West Africa. Afr Health Sci. 2016;16(3):690-7.

28. Speich B, Marti H, Ame SM, Ali SM, Bogoch II, Utzinger J, et al. Prevalence of intestinal protozoa infection among school-aged children on Pemba Island, Tanzania, and effect of single-dose albendazole, nitazoxanide and albendazole-nitazoxanide. Parasit Vectors. 2013;6(1):3.

29. Jejaw A, Zemene E, Alemu Y, Mengistie Z. High prevalence of Schistosoma mansoni and other intestinal parasites among elementary school children in Southwest Ethiopia: a cross-sectional study. BMC Public Health. 2015;15(1):600.

30. Tefera E, Belay T, Mekonnen SK, Zeynudin A, Belachew T. Prevalence and intensity of soil transmitted helminths among school children of Mendera elementary school, Jimma, Southwest Ethiopia. Pan Afr Med J. 2017;27:88. https://doi.org/10.11604/pamj.2017.27.88.8817. eCollection 2017..
31. Alamir M, Awoke W, Feleke A. Intestinal parasites infection and associated factors among school children in Dagi primary school, Amhara National Regional State, Ethiopia. Health. 2013;5(10):1697.

32. Tadesse $\mathrm{G}$. The prevalence of intestinal helminthic infections and associated risk factors among school children in Babile town, eastern Ethiopia. Ethiop J Health Dev. 2005;19(2):140-7.

33. Feleke BE. Nutritional status and intestinal parasite in school age children: a comparative cross-sectional study. Int J Pediatr. 2016;2016. https://doi.org/ 10.1155/2016/1962128. Epub 2016 Aug 30.

34. Haftu D, Deyessa N, Agedew E. Prevalence and determinant factors of intestinal parasites among school children in Arba Minch town, southern Ethiopia. Am J Health Res. 2014;2(5):247-4.

35. Gezehegn D, Abay M, Tetemke D, Zelalem H, Teklay H, Baraki Z, et al. Prevalence and factors associated with intestinal parasites among food handlers of food and drinking establishments in Aksum town, northern Ethiopia. BMC Public Health. 2017;17(1):819.

36. Gelaw A, Anagaw B, Nigussie B, Silesh B, Yirga A, Alem M, et al. Prevalence of intestinal parasitic infections and risk factors among schoolchildren at the University of Gondar Community School, Northwest Ethiopia: a crosssectional study. BMC Public Health. 2013;13(1):304.

37. Sadeghi H, Bakht M, Saghafi H, Shahsavari T. Prevalence of intestinal parasites in a population in Eghbalieh city from Qazvin Province, Iran. J Parasit Dis. 2015;39(2):126-9.

38. Mengist HM, Demeke G, Zewdie O, Belew A. Diagnostic performance of direct wet mount microscopy in detecting intestinal helminths among pregnant women attending ante-natal care (ANC) in east Wollega, Oromia, Ethiopia. BMC Res Notes. 2018;11(1):276

\section{Publisher's Note}

Springer Nature remains neutral with regard to jurisdictional claims in published maps and institutional affiliations.

Ready to submit your research? Choose BMC and benefit from:

- fast, convenient online submission

- thorough peer review by experienced researchers in your field

- rapid publication on acceptance

- support for research data, including large and complex data types

- gold Open Access which fosters wider collaboration and increased citations

- maximum visibility for your research: over $100 \mathrm{M}$ website views per year

At $\mathrm{BMC}$, research is always in progress.

Learn more biomedcentral.com/submission 